\title{
Software Libre para apoyo a los procesos educativos
}

Recibido: 15 Fed 2016 - Revisado: 30 Abr 2016

Aceptado: 30 May 2016 - Publicado: 30 Jul 2016

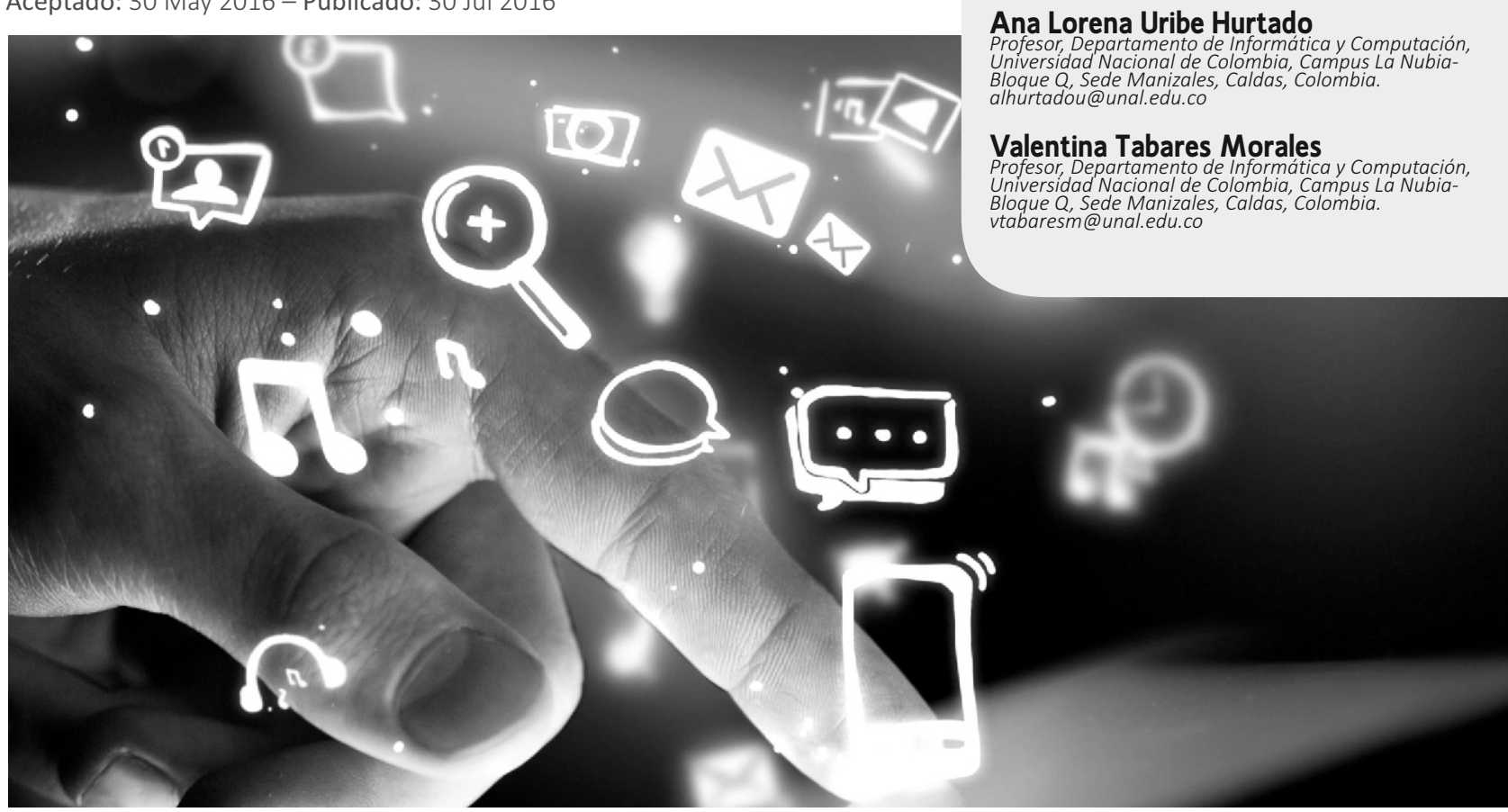

Resumen: Este artículo se orienta a mostrar el software libre como un camino relevante en los procesos educativos. Se muestran diferentes dimensiones que soportan esta afirmación, que van desde la filosofía del software libre, pasando por lo relacionado con los costos asociados y terminando con las alternativas en casi todos los campos del conocimiento. Se da especial importancia al beneficio ofrecido de potenciar competencias y valores que, casi siempre, en el software propietario, estarían restringidas; en particular, la posibilidad abierta para desarrollar a partir de trabajos previos de la comunidad, fortaleciendo capacidades tecnológicas y la creatividad y promoviendo la construcción colectiva altruista sin protagonismo. Finalmente, se presenta una propuesta para un Laboratorio Académico Genérico basado en Software Libre, cuyo aporte fundamental es mostrar que existen los elementos tecnológicos que permiten implementar, en diferentes ambientes, plataformas educativas completas para diferentes tipos de usuario.

Palabras clave: Plataformas educativas, software libre, tecnologías educativas.

Abstract: This article aims to show the Free Software as a relevant way in educative process. Different dimensions that support this assertion are presented. Ranging from the philosophy of Free Software, to the associated costs and ending with alternatives in almost all fields of knowledge. Special importance to the benefit offered to enhance skills and values that, almost always, in the proprietary software would be restricted, particularly the open possibility to develop from previous work of the community, strengthening technological capabilities and creativity and promoting construction altruistic collective. Finally, a proposal for an Generic Academic Laboratory based on Free Software, whose main contribution is to show that there are technological elements that allow you to implement complete educational platforms in different environments, for different types of user, is presented.

Keywords: . Educational platforms, free software, educational technologies 


\section{INTRODUCCIÓN}

Las Tecnologías de la Información y Comunicación (TIC) aplicadas a la educación han generado importantes cambios en la aplicación de modelos pedagógicos y en la didáctica en particular. El papel de la tecnología como mediador en el proceso enseñanza-aprendizaje es innegable, pero es importante destacar que lo que debe prevalecer es el proceso de enseñanza y aprendizaje y que las tecnologías deben ser mediadores para este. Como se expresa, desde los tiempo atrás, son más importantes las actividades humanas que los cables y equipos. Como en las demás esferas de la sociedad, la presencia de las TIC produce importantes e indiscutibles transformaciones.

Estas variaciones se presentan tanto en la gestión administrativa de la educación y en el salón de clases y allí, en todos los espacios: materiales educativos, laboratorios, accesos a los recursos bibliográficos y en general, a la información académica .

El problema abordado en este artículo está relacionado con una situación común. Para muchas instituciones de educación es casi inalcanzable la posibilidad de contar con una plataforma educativa que permita almacenar y ofrecer materiales educativos creados por los académicos, ofrecer diferentes actividades individuales y grupales y tener software científico para diferentes niveles y áreas, además de herramientas para el desarrollo de software.

Para este trabajo, el software no solo tiene aspectos tecnológicos, sino también filosóficos, sociales y económicos. En particular, toman importancia los elementos relacionados con la ubicación del estudiante respecto a estas herramientas, la disponibilidad de alternativas, pero además, los costos asociados con estas tecnologías. A partir de dichos aspectos, el enfoque propuesto se basa en la visión y en los desarrollos de la comunidad de software libre en las diferentes ciencias y disciplinas.
Este trabajo está organizado de la siguiente manera: En la sección dos, se recogen conceptos sobre las diferentes formas de ofrecer el software a la comunidad; en el siguiente aparte, se presentan algunas experiencias y proyectos de uso de software libre en programas educativos en distintos países; en la sección cuatro, se esbozan diferentes elementos que permiten ubicar el software en el ambiente educativo, para continuar con una propuesta integradora de laboratorio académico basado en software libre. Por último, se presentan las conclusiones y trabajos futuros.

\section{EL SOFTWARE Y EL ACCESO AL CONOCIMIENTO}

El acceso a la tecnología y en particular, al software está determinado por los propietarios o creadores de estos recursos. La visión que se tenga del conocimiento estará plasmada en los esquemas de distribución y participación de las aplicaciones desarrolladas.

A continuación, se exponen brevemente algunos conceptos relacionados con la forma de distribuir el software.

El Software Libre respeta la libertad de los usuarios y la comunidad en lo referente a ejecutar, copiar, distribuir, estudiar, modificar y mejorar el software. Esto significa que el Software Libre es una cuestión de libertad y no de precio. Las cuatro libertades esenciales son: a) La libertad de ejecutar el programa como se desea, con cualquier propósito. b) La libertad de estudiar cómo funciona el programa, y cambiarlo para que haga lo que se quiera. El acceso al código fuente es una condición necesaria para esto. c) La libertad de redistribuir copias para otros y d) La libertad de distribuir copias de las versiones modificadas a terceros. Esto le permite ofrecer a toda la comunidad la oportunidad de beneficiarse de las modificaciones. Un programa sea software libre no quiere decir que no sea comercial, puede estar disponible para el uso, la programación y la distribución comercial . 
Por otro lado: Open Source o código abierto se refiere al software que permite el acceso a su código fuente, posibilitando a otros usuarios modificar el software a su medida o aportar en la solución de las fallas probables. Para Open Source Iniciative (OSI) la filosofía del Open Source se basa en una cuestión de práctica y no de ética como la del Software Libre, se cree que al compartir el código el programa resultante tiende a ser de mejor calidad que el privativo. Los requisitos necesarios para considerar un programa código abierto son los siguientes: a) Libre redistribución: el software debe poder ser regalado o vendido libremente. b) incluir el código fuente. c) la redistribución de modificaciones debe estar permitida. d) las licencias pueden requerir que las modificaciones sean redistribuidas sólo como parches. e) sin discriminación de personas o grupos, todos incluidos, ni siquiera los usuarios comerciales se excluyen. f) deben aplicarse los mismos derechos a todo el que reciba el programa. g) La licencia no debe restringir otro software para que sea de código abierto.

El término freeware se refiere a software gratis, pero no necesariamente libre, permite redistribuir el programa pero con algunas restricciones como pueden ser no modificarlo ni venderlo o dar cuenta a su autor; Estos paquetes no son software libre,. El shareware, se distribuye gratis pero con uso limitado en el tiempo.

El software propietario o desde el enfoque ideológico, privativo, se refiere al antónimo de software libre y califica a cualquier programa informático que no es libre o que solo lo es parcialmente, sea porque su uso, redistribución o modificación está prohibida o porque requiere permiso expreso del titular del software.

Comercial y privativo no son la misma cosa. El software comercial es aquel que es desarrollado por una empresa como parte de su actividad comercial. La mayoría de los softwares comerciales son privativos, pero también existe software libre que es comercial y software privativo que no es comercial.

La Tabla 1 resume algunas de las características de estas diferentes posibilidades de ofrecer las aplicaciones.

Para la FSF (Free Software_Foundation), el software libre es más un movimiento social y el Open Source, un método de desarrollo; mientras que el Freeware, aunque su uso sea gratuito, es privativo. La Fig. 1 resume algunas de las posibilidades y características de la distribución de software.

Tabla 1. Características del software.

\begin{tabular}{|c|c|c|c|c|c|}
\hline & $\begin{array}{l}\text { Software } \\
\text { Libre }\end{array}$ & $\begin{array}{l}\text { Software } \\
\text { Open Source }\end{array}$ & Freeware & Shareware & $\begin{array}{l}\text { Software } \\
\text { propietario }\end{array}$ \\
\hline Sin costo & Casi siempre & Casi siempre & $\mathrm{Si}$ & $\begin{array}{l}\text { Por un } \\
\text { tiempo }\end{array}$ & Casi nunca \\
\hline $\begin{array}{l}\text { Código } \\
\text { fuente } \\
\text { abierto }\end{array}$ & $\mathrm{Si}$ & $\mathrm{Si}$ & No & No & $\begin{array}{l}\text { No o muy } \\
\text { restringido }\end{array}$ \\
\hline $\begin{array}{l}\text { Se puede } \\
\text { usar (U), } \\
\text { compartir } \\
\text { (C) } \\
\text { modificar } \\
\text { (M) }\end{array}$ & $U, C, M$ & $U, C, M$ & $\mathrm{U}, \mathrm{C}$ & U & U con pago \\
\hline $\begin{array}{l}\text { Carácter } \\
\text { Ideológico }\end{array}$ & $\begin{array}{l}\text { Libertad } \\
\text { conocimiento }\end{array}$ & $\begin{array}{l}\text { Libertad } \\
\text { conocimiento }\end{array}$ & $\begin{array}{l}\text { Compartir } \\
\text { Resultados }\end{array}$ & $\begin{array}{l}\text { Probar para } \\
\text { vender }\end{array}$ & $\begin{array}{l}\text { Propiedad } \\
\text { sobre } \\
\text { conocimiento }\end{array}$ \\
\hline
\end{tabular}




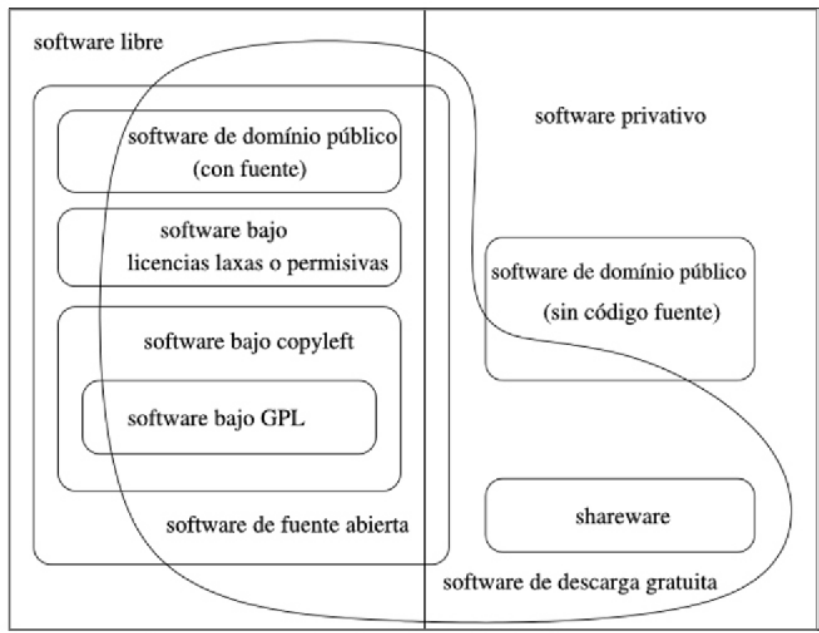

Figura 1. Categorias de software. Tomado de .

Para el movimiento por el código abierto, el asunto sobre si el software debiera ser de código abierto es una cuestión práctica, no ética;, el software que no sea libre es una solución ineficiente. Para el movimiento por el software libre, aquel que no es libre es un problema social y el software libre es la solución .

GNU es un acrónimo recursivo de GNU's Not Unix (GNU no es Unix) y se refiere a un proyecto creado en 1984 por Richard Stallman para obtener un sistema operativo completo, totalmente libre, basado en el sistema operativo Unix, para fomentar la libertad y cooperación en la creación de software.

\section{EL SOFTWARE LIBRE COMO ALTERNATIVA PARA LA GESTIÓN DE CONOCIMIENTO}

Una de las formas de reducir la gran brecha digital existente es permitir el acceso a la comunidad académica a la tecnología en las escuelas, colegios y universidades. El factor económico es uno de los principales impedimentos para que el acceso a la tecnología sea posible, por eso es importante explorar nuevas opciones que faciliten y permitan reducir dicha brecha, y el software libre cumple y ayuda de la mejor manera a lograr esta meta.
La comunidad de software libre y los proyectos auspiciados se han convertido en una alternativa real en las instituciones públicas y privadas; además, organizaciones reconocidas a nivel mundial apoyan e incentivan el uso y desarrollo de aplicaciones en diferentes ámbitos. mencionan algunos organismos a nivel mundial que ven en el software libre como una verdadera oportunidad de apropiación y potenciación tecnológica, especialmente para países en vías de desarrollo; entre ellos, la ONU y la Unesco.

En esta vía, hay experiencias en varias esferas de la sociedad: En Europa, el Partido Socialista Español (PSOE) impulsó la implantación de software libre en el sistema educativo español, creando un sistema centralizado para elaborar, recopilar y difundir materiales curriculares y de apoyo al profesorado en soporte informático y software libre. También en España, en Extremadura, el software libre es uno de los pilares en los que se apoya el proyecto global de acceso a la sociedad de la información; respaldan sus grandes inversiones en hardware con su producto local GNU/LinEx. Su éxito contagió al gobierno de Andalucía, que ya cuenta con su propia distribución de software libre basada en GNU/LinEx.

En Asia, es conocido el acuerdo firmado entre Japón, China y Corea del Sur para desarrollar de manera conjunta un nuevo sistema operativo basado en Linux como alternativa al dominante Microsoft.

En nuestro continente, también se ha avanzado en este camino. El gobierno argentino anunció la suspensión de la obligatoriedad legal de uso del sistema operativo Microsoft Windows; permitió que cualquier empresa o el mismo Estado pudiese mantener y actualizar sus sistemas simplemente contratando empresas locales, para combatir así el desempleo.

En Perú también se han hecho varias propuestas de ley de uso de software libre en las empresas del estado. 
Brasil es un caso de especial interés, con amplio apoyo gubernamental. El Proyecto Software Libre Brasil (http://softwarelivre.org), que es una iniciativa no gubernamental, reúne universidades, instituciones públicas (como la prefectura de Portoalegre o en el pasado, el Ejecutivo de Lula), empresas, grupos de usuarios, ONG, etc. Su principal objetivo es la promoción de uso de software libre como una alternativa económica y tecnológica. De este modo, estimula el uso de software libre; el proyecto invierte en la producción y calificación del conocimiento local a partir de una nueva postura que involucra la parte tecnológica en el contexto de construcción de un mundo con inclusión social e igualdad de acceso a los avances tecnológicos. En el área de la educación brasilera, uno de los principales programas del pasado fue el de inclusión digital, con metas ambiciosas y con recursos en proyectos que utilicen software libre.

En Colombia, son ya muchas las universidades y las empresas que están usando software libre en gran parte de sus actividades. La conectividad creciente le permitió al país ingresar a la comunidad de software libre mundial. Existen muchos grupos al interior de las universidades oficiales y privadas que agrupan a los usuarios de software libre; esto es algo que no se presenta en el ámbito del software propietario. Se han presentado propuestas de Proyecto de Ley de Software Libre en Colombia para incentivar el uso de este como mecanismo para fomentar el respeto a los derechos constitucionales de los ciudadanos y el desarrollo tecnológico de la nación. En Colombia, la Universidad del Valle soporta gran parte de su actividad académica en software libre.

Para Rajani (2003), durante los últimos años, el uso de software libre de código abierto (Free/Libre Open Source Software FLOSS) ha cobrado impulso, que ha sorprendido a sus partidarios y detractores por igual. En cuanto a las cifras, no sería exagerado decir que Internet es alimentado por FLOSS.

Dada la enorme variedad en el uso del software libre y su impacto económico muy significativo, las preguntas que surgen desde la perspectiva de la ayuda al desarrollo sostenible son: ¿ofrece el FLOSS una alternativa importante a los países en desarrollo para abordar los problemas cruciales, como el alivio de la pobreza, la democratización de la sociedad, la reducción del analfabetismo, la reducción de conflictos, el acceso a conocimiento? ¿FLOSS tiene el potencial de ayudar a cerrar la brecha digital? Según la opinión de los autores, las respuestas a la mayoría de las preguntas anteriores es un sí definitivo, pero con voluntad política y las fuerzas sociales necesarias para resolver los problemas; la tecnología es sólo una herramienta .

\section{SOFTWARE LIBRE Y AMBIENTE EDUCATIVO: UN LABORATORIO DE FORMACIÓN}

La libertad del software asume un rol de fundamental importancia en el ámbito educativo. El software libre permite a las instituciones cumplir con sus misiones fundamentales: difundir el conocimiento y enseñar valores a los estudiantes en beneficio de su comunidad. El código fuente y los métodos del software libre son parte del conocimiento humano y su acceso debe contribuir al desarrollo de la humanidad; mientras que para el software privativo, es conocimiento secreto y restringido, contrario la misión de las instituciones educativas.

Los estudiantes pueden usar las herramientas; interactuar con ellas en su configuración adquiere conocimiento de la forma en que construyó, puede mejorarlas, permiten la construcción creativa sobre ellas. El software apoya sus actividades, pero además, es fuente de conocimiento y un aliciente para la creatividad, condición para el desarrollo de un país.

Pero, adicional a la formación en valores que ofrece el software libre, la comunidad ha desarrollado herramientas que cubren todos los campos de conocimiento y en los diferentes niveles educativos. 
Ante esto, el software libre se constituye en una alternativa para la mayoría de ámbitos públicos y privados. Este conjunto de soluciones informáticas generadas bajo distintas licencias facilitan la reutilización de la experiencia (al estilo del conocimiento científico) y su uso generalizado y gratuito, además de fomentar el trabajo colaborativo. A decir de Stallman (2012), las escuelas tienen una misión social: enseñar a los alumnos a ser ciudadanos de una sociedad fuerte, capaz, independiente, solidaria y libre y deberían promover el uso de software libre al igual que promueven la conservación y el voto. Enseñando el software libre, las escuelas pueden formar ciudadanos preparados para vivir en una sociedad digital libre. Esto ayudará a que la sociedad entera se libere del dominio de las megacorporaciones.

El uso por sí de la tecnología no garantiza cambios significativos en los procesos, pero si estos van acompañados de estrategias fundamentadas, los logros son notables. Es posible ver los sistemas informáticos soportando el aprendizaje unidireccional, como un instrumento que ayuda a adquirir algunos conocimientos sin interacción de estudiante, limitando el aprendizaje. Pero, hay desarrollos en este campo que garantizan apoyar procesos bidireccionales donde el ambiente educativo se nutre con las interacciones de estudiantes y profesores; aquí, la tecnología es un facilitador para los procesos cognitivos. En el trabajo de , se presentan elementos claves para introducir las TIC en el aula de manera efectiva, y proponen distintas estrategias y enfoques cuya ventaja fundamental es que cumple con el objetivo primordial de todo sistema educativo: mejorar la formación del alumno y promover el éxito escolar.

En los procesos de formación, expresados por las declaraciones de objetivos de aprendizaje, estos se pueden organizar en una jerarquía de menos a más compleja. El mismo Bloom define su taxonomía cognitiva en seis niveles de complejidad creciente y cada nivel depende de la capacidad del alumno para desempeñarse en el nivel o los niveles precedentes. Por ejemplo, la capacidad de evaluar (el nivel más alto de la taxonomía cognitiva) se basa en el supuesto que el estudiante, para ser capaz de evaluar, tiene que disponer de la información necesaria, comprender esa información, ser capaz de aplicarla, de analizarla, de sintetizarla y, finalmente, de evaluarla. Estos diferentes niveles, incluso de más alto nivel pueden ser promovidos desde la concepción misma del software libre y de código abierto, y así, crear mentes proactivas, críticas y reflexivas.

Recogiendo la esencia de la discusión anterior, a continuación se presenta una propuesta de creación de un Laboratorio de Software Libre orientado a la formación de estudiantes de diferentes disciplinas. No se incluyen aplicaciones específicas, pero existen diversos trabajos que pueden ser consultados sobre esta materia. La Fundación del Software Libre (FSF) y la Unesco tienen un proyecto titulado "Directorio del Software Libre», en el cual presentan un catálogo de software libre que corren bajo sistemas operativos libres, particularmente, el Sistema Operativo GNU y I a s variantes de GNU/Lin ux .http://www.gnu.org/software/free-software-foreducation.html, aparece una lista de softwares con fines educativos o con posibilidad de uso en ambientes de enseñanza y aprendizaje, retomando otros trabajos, clasifican las herramientas de software en grupos; para el interés de este trabajo y con un papel específico en el fortalecimiento de competencias educativas, coinciden en los siguientes puntos:

(a) Herramientas para productividad en oficina: Permiten diversas aplicaciones, no están restringidas a un contenido disciplinario específico y su uso depende de la capacidad del usuario para sacarle provecho. Permiten fortalecer las capacidades lingüísticas, estéticas, espaciales y en el caso de aplicaciones concretas, se ve beneficiada la inteligencia lógica matemática. 
(b) Aplicaciones informáticas para actividades académicas o mediadores instrumentales educativos: Sirven como ayuda complementaria a la labor del profesor en el aula o incluso lo reemplazan en esta actividad. Entre ellas, tenemos tutoriales básicos, programas de simulación, laboratorios interactivos, sistemas tutoriales inteligentes.

(c) En el caso de los sistemas de ejercitación y simulación, las capacidades kinestésicas y motoras pueden recibir un buen empuje.

(d) Lenguajes de programación y herramientas de desarrollo. Es software para hacer software. Potencian y fortalecen la creatividad, la visión holística y la meta-cognición como un proceso de gran importancia en la educación.

(e) Herramientas de comunicación y trabajo en grupo: Procesos educativos soportados en www, correo electrónico, listas de correo, foros, chat, transferencia de archivos, audioconferencia, videoconferencia, etc. Dependiendo de su uso, apoyan el trabajo cooperativo y las relaciones interpersonales. Fortalecen las habilidades lingüísticas a nivel de lectura, escritura y formación de mensajes.

Se propone una arquitectura como la mostrada en la Fig. 2, donde se ofrezca acceso a servidores con software libre (que pueden estar en una o muchas máquinas), donde se ofrezcan servicios locales y remotos requeridos para impulsar las diferentes estrategias educativas.

En los casos que no se cuente con buenos canales de acceso a Internet, el esquema sigue siendo válido en el marco de una Intranet. Los clientes tendrían instalada una suite ofimática y herramientas de desarrollo, según las necesidades específicas, al igual que las aplicaciones requeridas para la conexión a los servidores. Sin duda, hay diferentes tipos de usuarios, por lo cual, en los clientes, debe disponerse de las herramientas específicas para cada uno.

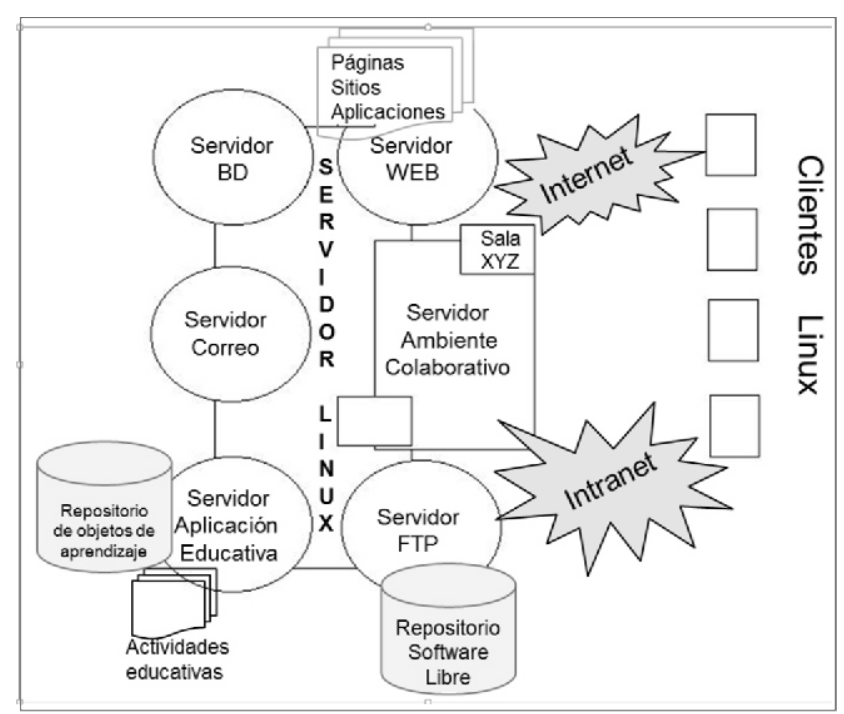

Figura 2. Arquitectura para soporte a la educación basada en software libre.

En ocasiones, es posible que no se cuente con la disponibilidad de los recursos en hardware para optar por una arquitectura que apoye el modelo de la Figura 2. Es viable optar por alternativas en el lado del servidor, como los clúster en Linux y las estaciones con baja configuración se pueden adaptar para usar los servidores a través de accesos virtuales o tecnologías más recientes, que permiten compartir el escritorio entre máquinas de una red, así, los alumnos pueden ver el monitor del profesory a la inversa.

Para el caso de instituciones de educación básica y media, como aspecto especial, se puede tener en cuenta el sistema operativo Linux, que incluye muchas de las aplicaciones en las diferentes distribuciones; entre ellas Edubuntu, cuyo objetivo es armar un sistema que contiene todo lo mejor del software libre disponible en la educación y que sea fácil de instalar y mantener. Sin embargo, la instalación de las distribuciones Linux, en los casos que forme parte de las actividades propuestas en un curso, sin duda exige de la puesta en funcionamiento de múltiples mecanismos cognitivos, lo que la vuelve en sí misma una importante actividad educativa. Por otro lado, como plataforma de trabajo, crea condiciones 
especiales para diferentes actividades de enseñanza (individuales o grupales) que facilitarían el aprendizaje conceptual, procedimental y actitudinal.

Para cada uno de los componentes de la arquitectura, se cuenta con diferentes productos en la comunidad open source como reemplazo de casi cualquier aplicación comercial. Por otra parte, existen sitios específicos donde se pueden encontrar aplicaciones académicas y ofimáticas libres y abiertas para diferentes plataformas, entre ellos: www.linuxlinks.com y www.linux.com.

\section{CONCLUSIONES}

Este trabajo coloca al software libre como una verdadera herramienta para el soporte de la educación en los diferentes niveles y desde diferentes estrategias educativas, que incluso supera las posibilidades en los sistemas de enseñanza basado en productos propietarios. Por otro lado, promueve y expone los valores del Proyecto GNU en las escuelas: Libertad, Comunidad y Cooperación.

Se presenta una propuesta de Laboratorio para la docencia basado en software libre, cuyo aporte fundamental es mostrar que existen los elementos tecnológicos que permiten implementar en diferentes ambientes, plataformas educativas completas para diferentes tipos de usuario.

TRABAJO FUTURO se prevé el diseño e implementación de una suite que contenga diversas herramientas y que permita ser parametrizada para una fácil instalación y se adecúe a los currículos específicos. Por otra parte, como plataforma de trabajo, crea condiciones especiales para diferentes actividades de enseñanza (individuales o grupales) que facilitarían el aprendizaje conceptual, procedimental y actitudinal.

AGRADECIMIENTOS Este trabajo se enmarca dentro de las propuestas generadas en el Laboratorio de
Software Libre del Departamento de Informática y Computación de la Universidad Nacional de Colombia, sede Manizales.

\section{REFERENCIAS}

Bloom, B. S. (1956). Taxonomy of the Cognitive Domain. Educational Psychology Interactive, 1-4.

Dávila Sguerra, M. (2013). El software libre en Colombia. Popayán: Colombia Digital. Recuperado de: http://gluc.unicauca.edu.co/archivos/referencia/El_s oftware_libre_en_Colombia.pdf

FSF Free_Software_Foundation. (2014). Diferencias entre Software Libre, Open Source y Freeware. Recuperado de: https://lignux.com/diferencias-entresoftware-libre-open-source-y-freeware/

FSF \& Unesco. (2015). Free Software Directory. Recuperado de: http://directory.fsf.org/

Gnu.org (2004). Categorías de software libre y software que no es libre. Recuperado de: http://www.gnu.org/philosophy/categories.es.html Gnu.org. (2011). Free Software and Education. Recuperado de:

http://www.gnu.org/education/education.en.html

González, G., Méndez, Y., Jiménez, J. E., Vasques, K., \& Checa, A. (2007). Software libre en la educación. Recuperado de:

http://www.colombiaaprende.edu.co/html/mediatec a/1607/articles-108475_archivo.pdf

Harvey, C. (2010). 50 Open Source Tools That Replace Popular Education Apps. Datamation, 8-9.

Maican, C., \& Lixandroiu, R. (2016). International Journal of Information Management A system architecture based on open source enterprise content management systems for supporting educational institutions. International Journal of Information 


\section{Ingenierías:r.:}

$\mathrm{M}$ a n a g e m e n t, $36(2), 207-214$. http://doi.org/10.1016/j.ijinfomgt.2015.11.003

Nieto Moreno de Diezmas, E., \& Graells Marquès, P. (2015). La mejora del aprendizaje a través de las nuevas tecnologías y de la implantación del currículo bimodal. Multiárea: Revista de Didáctica, 7, 7-30.

Nuñez, I., \& Govín, G. (2005). Propuesta de clasificación de las herramientas - software para la gestión del conocimiento. ACIMED, 2005, 13(2). Recuperado de: http://eprints.rclis.org/6472/
Rajani, N. (2003). Free as in Education. Significance of the Free / Libre and Open Source Software for Developing Countries (OneWorld F).

Randy, B. (1999). Educational technology planning a framework. Victoria, B.C.: Centre for Curriculum,.

Stallman, R. (2009). Why Open Source misses the point of free software. Communications of the ACM, 52(6), 31. http://doi.org/10.1145/1516046.1516058

Stallman, R. (2012). On-line education is using a flawed Creative Commons license. Recuperado de: https://stallman.org/articles/online-education.html 